\title{
Quand le BIM met la maquette à l'épreuve du numérique
}

\author{
Émilien Cristia ${ }^{1,2}$, Pierre-Paul Zalio ${ }^{2}$, et François Guéna ${ }^{1}$ \\ ${ }^{1}$ MAP-MAACC, ENSA Paris La Villette, 75019 Paris, France \\ ${ }^{2}$ IDHES, ENS Paris-Saclay, 94230 Cachan, France
}

\begin{abstract}
Résumé. Cet article cherche à initier une réflexion théorique, empirique et sociologique sur le concept de maquette numérique considéré dans le cadre de projets BIM. Ainsi, nous considérons dans un premier temps la maquette physique qui, par effet miroir, nous sert à identifier certaines caractéristiques théoriques et empiriques inhérentes à la maquette numérique BIM. Puis, portés par l'intérêt de considérer l'hétérogène diversité des acteurs qui lui sont rattachés, nous approfondissons notre description par le prisme de la sociologie. En particulier, ceci nous permet d'envisager un rapprochement entre la maquette numérique BIM et le concept d'objetfrontière.
\end{abstract}

Mots-clés. Théorie de l'Acteur-Réseau, BIM, Maquette Physique, Maquette Numérique, Objet-frontière.

\begin{abstract}
This article initiates a theoretical, empirical and sociological reflection on the concept of digital mockup considered in BIM projects. By comparing these two types of mockup, some theoretical and empirical characteristics of a digital mockup will be suggested. Then, the article assumes some sociological considerations as the heterogeneous diversity of actors involved with this new artifact to complete the description. This leads us to link the concept of digital mockup with the concept of boundary object.
\end{abstract}

Keywords. Actor Network Theory, BIM, Physical mockup, Digital mockup, Boundary-Object.

\section{Introduction}

À travers le nouvel artefact numérique BIM, la maquette numérique qui lui est associée affecte l'ensemble du monde de la construction et la quasi-totalité des acteurs du projet. Les effets de la construction collective de cette maquette numérique sont multiples : nouvelle importance prise par la modélisation géométrique, mise en place de nouveaux processus métiers, automatisation de la gestion des documents, apparition de nouvelles professions ou encore émergence d'une collaboration numériquement assistée. Si l'étude de ces effets sur l'ensemble des acteurs du projet motive actuellement une recherche doctorale, cet article se 
focalisera sur une caractérisation et une description possible de la maquette numérique selon différents points de vue. Nous proposons dans un premier temps de tisser une comparaison conceptuelle et empirique entre les objets de maquette physique ${ }^{1}$ et de maquette numérique. La multiplicité des éclairages considérés sur ces objets cherche à faire émerger un champ de caractéristiques inhérent à la maquette numérique BIM et à en identifier les points de rupture avec son homologue physique. Nous mobiliserons ensuite la sociologie des techniques pour proposer une seconde lecture de cette maquette numérique, notamment afin de considérer l'ensemble des acteurs du projet. Dans ce nouveau contexte, nous proposons de réinvestir le concept d'objet-frontière comme outil de caractérisation de la maquette numérique. Ces deux approches, bien que dissociées, entendent défricher de façon parallèle, mais complémentaire de nouvelles pistes possibles d'investigations autour de la maquette numérique BIM, reliant concepts théoriques, observations empiriques et sociologie des techniques.

\section{Vers un nouveau concept de maquette : maquette physique vs maquette numérique}

\subsection{Traçabilité d'une tâche}

Les dictionnaires courants nous apprennent que le nom de maquette renvoie à sa racine italienne macchietta diminutif de macchia dont l'étymologie latine macula désigne une macule, soit, par transposition une tâche. Une tache peut être perçue comme une marque ou un signe témoignant d'une expérience ou d'une action passée, réalisée volontairement ou non. Daniel Estevez considère ainsi la maquette physique comme une trace inhérente à la recherche du projet qui résulte d'un " processus continu et ininterrompu d'actions et de réflexions entrecroisées " (Estevez, 2014a, p. 79). Lorsque Giorgio Vasari évoque les premières maquettes en mie de pain du Duomo réalisées par Filippo Brunelleschi, il fait référence à la perte d'une trace mémorielle du projet. L'histoire de cet édifice sera d'ailleurs marquée par une seconde maquette (en bois cette fois) qui, léguée aux Florentins après la mort de son auteur, permit l'achèvement de l'ouvrage. La maquette physique se veut ainsi chargée d'une certaine mémoire, que ce soit à travers les traces du processus qui l'a vue naitre ou bien l'état d'une connaissance finalisée qu'elle incarne.

L'analogie avec la maquette numérique semble peu aisée car sa nature numérique induit une construction et une implémentation par ajout/suppression de données. Or ces actions ne laissent en général que peu de traces visuellement identifiables. Aucun dépôt de colle ou empreinte de moule ne peut témoigner qu'un autre élément était là avant et a fait partie du processus d'élaboration de ladite maquette numérique. S'il est théoriquement possible de revenir à des états antérieurs d'élaboration de certains éléments d'une maquette numérique, l'entreprise apparait laborieuse et de peu d'intérêt, surtout lorsque l'on connait les efforts que requiert la lecture d'un fichier ou d'une sauvegarde issue de versions devenues obsolètes pour un logiciel. Catherine Deshayes, qui s'intéresse à l'acte de conception constate aussi une vraie difficulté inhérente à la traçabilité d'objets construits au moyen de logiciels informatiques, incluant la maquette numérique BIM (Deshayes, 2013, p. 127). Une maquette numérique, par sa nature et son mode de construction, semble donc en rupture avec la valeur sémantique de trace qu'elle porte en elle. Les raisons de cette difficile traçabilité peuvent s'expliquer par l'instabilité qui caractérise ce nouvel objet. En

${ }^{1}$ La maquette physique est ici entendue comme la traditionnelle maquette architecturale qui, jusqu'à l'apparition du BIM, semblait se passer d'adjectif pour ne pas être confondue. 
effet, une fois achevée, la maquette physique se conçoit comme l'instrument d'une connaissance stabilisée, alors que la maquette numérique BIM ne semble jamais se stabiliser car elle ne cesse d'évoluer tout au long de la vie de l'ouvrage qu'elle incarne - et c'est bien là tout son intérêt.

\subsection{Considération dimensionnelle}

Avant d'examiner l'intérêt du relatoscope inventé au début des années soixante-dix par Martin Schultz Van Treeck (figure 1) qui constitue probablement l'un des premiers dispositifs de construction d'image immersive à partir d'une maquette physique, François Loyer rappelle que, pour l'architecte « la maquette [est] le seul outil de création capable de rétablir et conserver à chaque moment du projet la troisième dimension » (Loyer, 1974, p. 56). Par comparaison, les autres outils traditionnels de représentation (plan, coupe, perspective ou axonométrie) mobilisés par l'architecte, s'expriment exclusivement en deux dimensions. Il convient d'ailleurs de rappeler que leur lecture s'effectue de manière théorique, autorisant notre œil à franchir planchers, murs et cloisons sans même s'en rendre compte. Ces moyens de représentation parviennent cependant à rétablir certaines notions inhérentes à la tridimensionnalité du projet : volume, ensoleillement, luminosité, profondeur grâce à une large palette d'artifices et de codes graphiques (épaisseur de traits, ombres projetées, pointillés, textures), considérablement facilités par le développement des logiciels graphiques. La maquette physique, vue comme objet tridimensionnel, semble quant à elle se suffire à ellemême pour être interprétée, sans avoir à faire appel à des conventions ou à des artifices graphiques autres que sa propre matérialité.

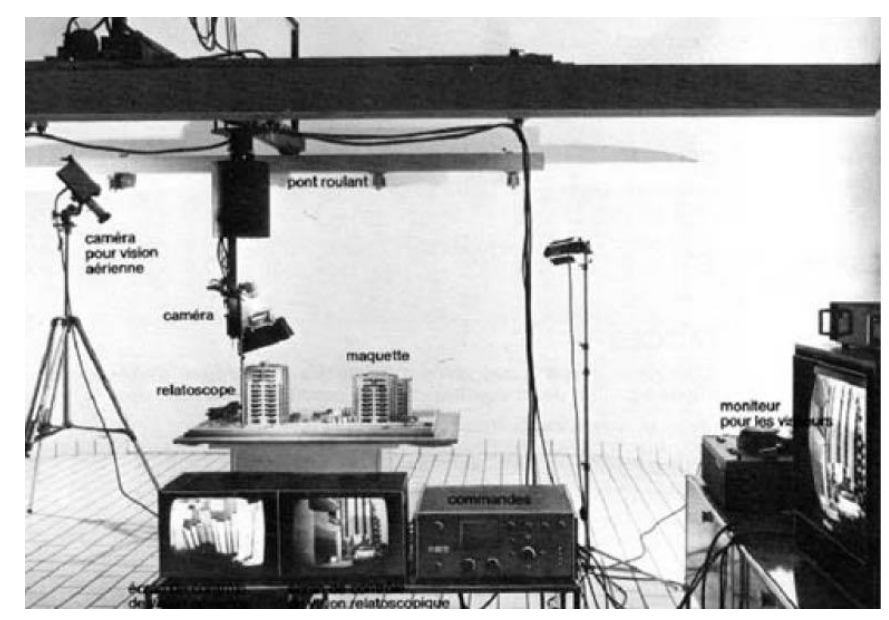

Figure 1. Représentation du dispositif du relatoscope.

Instinctivement, la question tridimensionnelle rapportée à la maquette numérique BIM repose essentiellement sur la représentation des différentes modélisations géométriques qui lui sont rattachées (Halin et Gallas, 2016, p. 89). D'ailleurs, l'assimilation de la notion de maquette numérique à celle de modèle ${ }^{2}$, avec lequel elle n'est pas sans lien, n'est pas rare (Girard, 2014 ; Halin et Gallas 2016). Ce glissement sémantique facilite les ponts avec le

${ }^{2}$ Le modèle dont on parle ici caractérise les modèles informatiques qui n'ont plus rien à voir avec ce que la théorie de l'architecture a pu construire depuis les modèles d'Andrea Palladio jusqu'aux modèles de villes d'Aldo Rossi. 
monde informatique, surtout lorsque la maquette numérique BIM est interprétée comme un outil de conception, de représentation ou encore de simulation. Or, la zone d'influence de la maquette numérique ne semble pas se restreindre à ces domaines uniquement. En effet, la mobilisation d'une maquette numérique BIM tout au long des phases du projet ainsi que ses interactions avec les multiples acteurs, nous autorise à imaginer des visées plus larges (synchronisation des pratiques, gestion des informations, coordination d'acteurs hétérogènes, organisation du travail, etc.). Même en acceptant d'omettre quantité d'informations, paramètres, fiches techniques et autres tableaux, pour ne considérer que la représentation des différents modèles géométriques qu'elle intègre, il demeure tout de même difficile d'assimiler la maquette numérique BIM à un objet tridimensionnel. En effet, si l'écran d'ordinateur reste le support privilégié d'interactions et de visualisations possibles de ces modèles, il se réduit à la construction d'images bidimensionnelles uniquement. Or, ces images ou « visibilités calculées » (Coulais, 2015) véhiculent parfois l'idée fausse d'une tridimensionnalité de l'objet représenté. La maquette numérique demeure en effet un objet numérique virtuel (Chalon, 2004, p. 19) et donc sans dimensions physiques qui puissent être quantifiées. On notera d'ailleurs que la maquette numérique se conçoit juridiquement comme objet immatériel $^{3}$ (Lefauconnier, 2017, p. 95) car numérique, confortant ainsi l'idée qu'elle constitue un objet adimensionnel. Afin de ne pas réduire la maquette numérique BIM aux éléments géométriques qui la composent, il n'est ainsi pas rare de la voir définie comme une base de données généralisées. Cependant utiliser la terminologie de base de données, plutôt que celle de maquette numérique, semble vider l'objet d'une grande partie de son enveloppe conceptuelle.

\subsection{Expérience sensible de la maquette}

La maquette physique peut exister sous des types ou familles variables (Estevez, 2014b) et être mobilisée pour des besoins divers : étude de conception, présentation de concours ou encore détail appuyant une solution constructive. Selon les besoins, les maquettes physiques convoquent ainsi un langage esthétique et sensoriel extrêmement varié. On gardera à l'esprit les maquettes de Antoni Gaudí en chiffons et fils de fer ou les maquettes de détail en bois et métal prisées par Renzo Piano en passant par les maquettes expérimentales de papier et de carton de récupération de Yona Friedman (figure 2). Chacune de ces maquettes physiques prouve, à travers leur matérialité et la diversité de leurs expressions, la construction d'une multiplicité de langages permettant d'asseoir un discours architectural singulier. Par ailleurs, pour l'architecte, ces maquettes physiques incarnent symboliquement son autorité $^{4}$, matérialisent son acte créateur et le définissent en tant qu'auteur. Elles constituent aussi des objets presque inviolables, voire sacrés, et garantissent à l'architecte, à travers leurs manifestations, une certaine considération de ses collaborateurs. De plus, Leon Battista Alberti considère la maquette physique comme un objet d'expérience sensible. L'expérience entendue comme sensible l'est doublement : à savoir au cours de la réalisation de l'objet et lors de son examen. La maquette physique fait ainsi appel à nos sens, bien évidement la vue et plus particulièrement le toucher, mais nous pourrions également

\footnotetext{
${ }^{3}$ La caractéristique immatérielle des objets numériques serait selon Christian Girard (Girard, 2014) en passe de devenir obsolète, en particulier face au développement de certaines technologies F2F.

${ }^{4}$ Il convient de rappeler que les noms d'auteur et d'autorité partagent une racine latine commune : auctor.
} 
imaginer qu'elle puisse solliciter l'ouïe, l'odorat, ou le goût selon les éléments dont elle est composée ainsi que son mode de réalisation ${ }^{5}$.
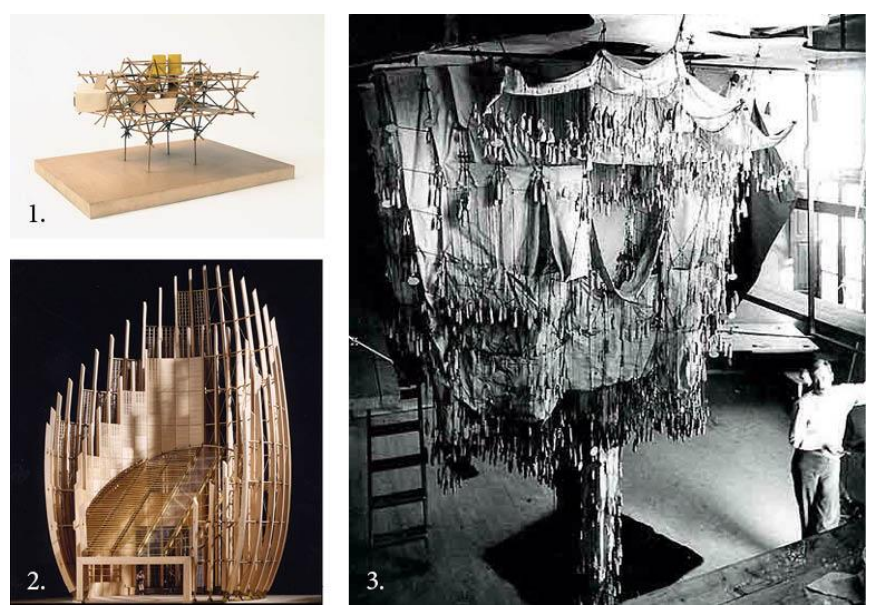

Figure 2. 1. Maquette utopique de Yona Friedman. 2. Maquette en bois du projet du centre culturel Tjibaou de Renzo Piano. 3. Maquette de chiffons et fils d'acier réalisée pour la Sagrada Família de Antoni Gaudí.

L'expérience sensible de la construction d'une maquette numérique se limite pour l'instant au couplage de certaines de ses représentations, avec des dispositifs de réalité augmentée ou d'environnement virtuel. En revanche, quelle autre expérience que la simple activité computationnelle pourrait-on identifier pendant la construction de l'objet? Or, l'utilisation généralisée des ordinateurs a certainement banalisé l'acte de computation et rendu en même temps la recherche d'une spécificité sensorielle ou expérientielle singulière plus difficile à déceler. D'autre part, considérer que le numérique n'a pas su créer de nouveaux langages esthétiques et sensoriels serait certainement une erreur : des artistes et architectes tels que Marcos Novak ou Zaha Hadid ont depuis longtemps démontré le contraire. En revanche, identifier dans les représentations qu'une maquette numérique BIM peut prendre la présence d'un langage esthétique et sensoriel semble aujourd'hui encore compliqué. De plus, dès l'instant où la maquette numérique BIM devient un objet partagé et distribué entre les différents acteurs du projet, elle ne peut plus être considérée comme le produit d'un concepteur unique. Le langage des représentations graphiques que l'on peut avoir d'une maquette numérique BIM (ou du modèle géométrique qui lui est associé) semble ainsi relativement homogène voire standardisé (figure 3). De nombreux architectes se plaignent aussi du simple manque de liberté graphique (et donc des langages) qu'autorisent les nouveaux logiciels de modélisation géométrique sémantique tels que Revit ou ArchiCAD. Ceci nous laisse donc penser que l'expérience sensible dans la construction d'une maquette numérique semble être atténuée et que l'élaboration collective de l'objet tendrait à uniformiser son langage d'expression.

${ }^{5}$ L'exposition Ma quête d'architecture, Maquette d'architectures organisée en 1993 au Pavillon de l'Arsenal a pu mettre en avant à travers près de 400 maquettes la diversité des types, formes, matériaux, langages, et expressions dont elles peuvent faire preuve. Pour quelques exemples on pourra consulter l'abécédaire du même nom publié par Clarisse (Clarisse, 1997). 

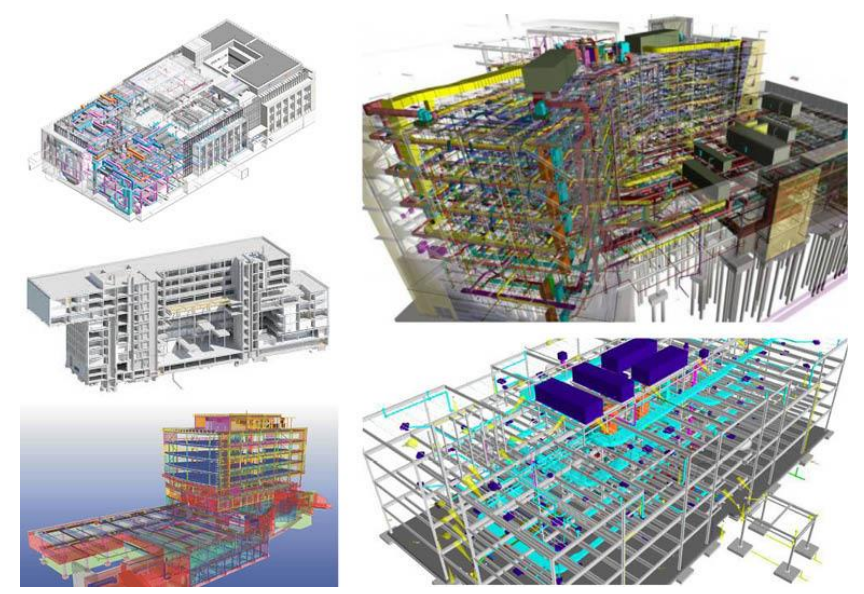

Figure 3. Exemple de diverses représentations proposées par Google Image avec les mots clés «maquette numérique BIM ».

\subsection{Singularité, multiplicité et objectifs}

Il est clair que la réalisation d'une maquette physique peut s'effectuer de manière individuelle. Cependant, cette maquette n'a pas souvent vocation à être isolée ou singularisée et constitue rarement un objet unique «fignolé, poncé, enjolivé » (Alberti, 2004, p. 99). D’ailleurs, D. Estevez rappelle que « la pertinence des maquettes est ainsi reliée à leur prolifération » (Estevez, 2014a, p. 79). La puissance d'utilisation de la maquette physique se dégage donc par un caractère souvent pluriel, sériel, qui lui confère une visée de mise en relief de la différence. On entrevoit d'ailleurs à travers l'ethnographie que fait Albana Yaneva de l'agence OMA (Yaneva, 2009), certaines pratiques projectuelles qui recherchent justement l'épuisement de solutions par la surabondante construction de maquettes en mousse bleue ${ }^{6}$ (figure 4).

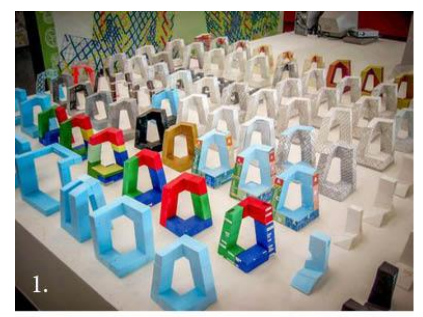

"The foam in the office intensifies the sense of imediate living ; models are objects of intense admiration, of thought provocation."

2.

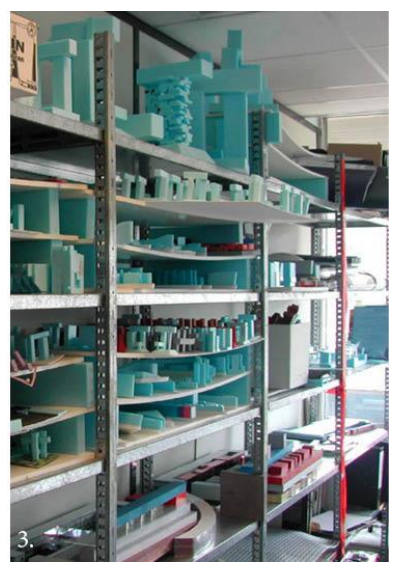

Figure 4. Exposition de maquettes du projet de la tour CCTV. 2. Citation de A. Yaneva (Yaneva, 2009, p.59). 3. Étagères de l'agence OMA rassemblant les maquettes de divers projets.

${ }^{6}$ OMA a d'ailleurs un temps construit une partie de son image sur cette fameuse mousse bleue. On la retrouve ainsi dans de multiples présentations de leurs projets, mais aussi, dernièrement, comme mobilier scénographique pour la célèbre marque Prada (voir Prada Men's Show, 2011). 
Bien évidemment, l'idée de construire une maquette numérique BIM de façon individuelle va totalement à l'encontre des objectifs qu'on lui prête. Et si l'on parle souvent de la maquette numérique comme d'un objet singulier, dans la pratique, il peut cependant coexister, pour un même projet BIM, une quantité affolante d'entités désignées comme maquettes numériques. Il existe ainsi tout au long du projet de multiples maquettes numériques construites et enrichies par différents acteurs qui, en s'associant les unes aux autres, génèrent théoriquement $L a$ maquette numérique du projet ${ }^{7}$. Pour un projet comme celui de l'ENS Paris-Saclay (BIM d'or 2015$)^{8}$, on comptabilise déjà en phase chantier, un an avant la livraison du bâtiment, plus de cent quatre-vingts maquettes numériques réparties entre les différents acteurs. Au total, depuis le début du projet, ce sont près de sept cents maquettes numériques - représentant environ 125 Go - qui ont été produites ! Dans ce cas précis, il reste évidemment impossible (et sans grand intérêt) de vouloir toutes les combiner pour ne faire face qu'à une seule et unique maquette numérique BIM. Cette surabondance de maquettes est due d'une part à des limites technologiques (incapacité des logiciels à gérer de multiples fichiers très volumineux), d'autre part au temps d'adaptation nécessaire aux différents acteurs du projet pour collaborer à l'échelle “BIM niveau trois ". Paradoxalement, maquettes numériques et physiques bien que toutes deux multiples constituent deux objets dont les visées sont largement différenciées. Premièrement, la maquette numérique BIM se distingue de la maquette physique comme étant un objet destiné à être construit collectivement, ce qui n'est pas un prérequis pour l'élaboration d'une maquette physique. Puis il faut reconnaître que le caractère multiple de la maquette numérique n'est en réalité que le résultat temporaire d'une innovation encore en cours de stabilisation. En effet, en théorie, un projet BIM réalisé dans des conditions optimales (BIM niveau 3) doit pouvoir permettre l'élaboration d'une unique maquette numérique sur laquelle tous les acteurs travaillent conjointement. C'est pourquoi la multiplicité des maquettes numériques que l'on rencontre dans les projets BIM n'est en rien comparable, ni dans l'intérêt pratique, ni dans les visées conceptuelles, à la prolifération des maquettes physiques évoquée par D. Estevez.

\subsection{Je maquette, tu maquettes, il maquette...}

Comme nous l'avons esquissé jusqu'à présent, la maquette numérique occupe une place centrale au sein d'un projet BIM. D'ailleurs, plusieurs observations ${ }^{10}$ effectuées au sein de différents projets BIM dont le projet de l'ENS Paris-Saclay, nous permettent de rapidement identifier la construction d'un langage commun, construit autour du champ lexical de la maquette (numérique). Dans les projets BIM, on constate effectivement un nouvel usage de terminologies liées à la maquette. Ainsi les termes «maquettage» ou « maquetter» ren-

\footnotetext{
${ }^{7}$ On rencontre parfois la dénomination de maquette métier pour désigner les maquettes produites par des corps de métier distincts.

${ }^{8}$ Le projet est notamment récompensé pour son investissement et son implication dans une démarche full-BIM ou de co-modélisation multidisciplinaire autour d'une maquette synchrone. Il est prévu une livraison du bâtiment avec DOE (Dossier des Ouvrages Exécutés) numérique et la maîtrise d'ouvrage envisage l'exploitation-maintenance du bâtiment grâce aux données générées par le mode projet BIM.

${ }^{9}$ On retrouve dans la littérature spécialisée quatre niveaux de maturité BIM échelonnés de zéro à trois. Le niveau trois correspond à un niveau BIM intégral, mais n'est pas encore atteint à ce jour dans la pratique.

${ }^{10}$ Le matériel empirique mobilisé s'étend de l'observation au sein de réunions de synthèse à la réalisation d'entretiens auprès d'acteurs de la maitrise d'œuvre et d'ouvrage, en passant par la mise en place d'expertises ou encore d'immersions au sein d'équipes du projet.
} 
voient par exemple à alimenter en information une maquette numérique. L'opération consiste soit à modéliser géométriquement un objet, soit à implémenter certaines des données ou paramètres de l'objet (matériaux, coût, fiche technique associée). D'autre part, on observera que la maquette numérique est ainsi tour à tour mise à jour, publiée, validée ou refusée par le BIM manager qui vérifiera l'intégrité des données qu'elle partage (géolocalisation du modèle, dénomination, respect de la charte) puis diffusée sur la GED ${ }^{11}$. L'identification de ces éléments langagiers au sein de projets BIM marque une nouvelle appropriation et un nouvel usage de la notion de maquette par les différents acteurs. Ceci vient évidemment rompre avec l'usage traditionnel qui pouvait être entendu à travers l'utilisation du champ lexical de la maquette (physique) et dénote une mutation dont on entre-aperçoit déjà certains effets sociologiques. Dans le contexte BIM, le terme de maquette ne renvoie ainsi plus à l'objet physique cantonné à l'atelier de l'architecte ou à l'étagère du maître d'ouvrage, mais devient un nouvel objet circulant entre les différents acteurs et construisant de nouveaux réseaux d'interactions.

Cette première partie nous a permis de caractériser la maquette numérique BIM comme un objet immatériel, adimensionnel, à la traçabilité limitée et dont le caractère sensible ou expérientiel inhérent à son processus de fabrication semble peu évident à déceler. De plus, c'est un objet évolutif, aujourd'hui pluriel et dissocié car partagé à l'ensemble des acteurs du projet, mais dont l'objectif une fois stabilisé serait d'être unique. De telles caracté-ristiques auraient pu nous laisser croire à une dilution progressive de l'objet dans le projet, mais sa réalité sociale démontre au contraire son omniprésence. Les projets BIM semblent ainsi construire de nouveaux référentiels conceptuels et théoriques autour de la maquette numérique dont il convient de poursuivre l'investigation.

\section{De l'objet au projet}

Vouloir essayer de rendre compte de la complexité à la fois technique et sociale dont fait preuve la mise en place d'une maquette numérique au sein d'un projet BIM, nous invite naturellement à opter pour un point de vue issu de la sociologie des techniques. La maquette numérique, perçue comme objet technique, entretient de multiples rapports entre les différents acteurs et objets (réels ou virtuels) redéfinissant les normes du projet. La Théorie de l'Acteur-Réseau ${ }^{12}$ (ANT) a su démontrer sa capacité à décrire et analyser la construction d'objets techniques ou d'innovations (Latour, 1992). Cette théorie considère que ces objets ont la capacité d'enrôler des acteurs en suivant la trame d'une coopération sociotechnique. Pour le cas de la maquette numérique, l'objet n'est pas directement donné ou stabilisé (Akrich, 1987), mais il est encore "en train de se faire ». Et c'est en particulier en s'attachant à suivre les fils du réseau d'interaction et de coopération qui mettent en jeu les effets d'enrôlement d'acteurs que la description de l'objet en construction est permise. L'utilisation de concepts théoriques tels que celui d'objet intermédiaire ou d'objet-frontière ${ }^{13}$ a reçu (et continue de recevoir) un certain succès dans les champs qui interrogent des activités de conception telles que le design (Bassereau, 2010) ou encore l'industrie et l'ingénierie (Jeantet, 1998 ; Boujut, 2003). A l'image de nouveaux objets

\footnotetext{
${ }^{11}$ Gestion Électronique des Documents.

12 L'Actor-Network Theory (ANT), ou sociologie de la traduction, est développée au début des années 80 par des chercheurs du Centre de Sociologie de l'Innovation (CSI) (dont Madeleine Akrich, Michel Callon et Bruno Latour). Cette théorie considère que les humains et non-humains ne constituent pas deux mondes distincts, mais sont étroitement imbriqués et interagissent entre eux.
}

${ }^{13}$ Le concept d'objet-frontière est construit et développé par Star et Griesemer (Star et Griesemer, 1989) et puise son essence de l'objet intermédiaire duquel il se distingue (Vinck, 2009 ; Star, 2010). 
numériques tels que les $\mathrm{SIG}^{14}$ (MacEachren, 2001), on peut considérer que la maquette numérique BIM fonctionne elle aussi comme un objet-frontière au sens où elle autorise « des éléments structurels (dans ou associés à l'objet ou à un ensemble d'objets) [à être] partiellement communs à plusieurs mondes sociaux » (Vinck, 2009, p. 66). Le concept d'objet-frontière permet donc de replacer la maquette numérique BIM à l'intersection des différents mondes sociaux dans lesquels elle évolue tout en permettant l'étude des activités des acteurs qui la mobilisent.

Dans le contexte d'un projet BIM, on observe en effet que la maquette numérique est le support quotidien d'échanges, de malentendus, de mises au point et de négociations portant sur le projet. Prenons ainsi l'exemple d'un architecte qui souhaite faire comprendre à une entreprise chargée de la $\mathrm{CVC}^{15}$, où il souhaite voir passer d'importantes gaines d'aération visibles depuis un espace central. Il modélisera à travers la maquette numérique l'encombrement et la position exacte où il souhaite les voir apparaître. Sur la base de cet échange et de la visualisation de sa maquette métier, une négociation débute mettant en jeu le sous-dimensionnement des gaines modélisées en fonction du débit requis, la nécessité de changer de produit engendrant un coût supplémentaire, l'impossibilité de notifier de nouvelles réservations au gros œuvre, ou encore l'éventuelle mise en place d'une FMT ${ }^{16}$. Ces interactions conduisent donc des professions aux frontières différenciées par des intérêts économiques, des responsabilités contractuelles ou des champs de compétences, à se coordonner autour de l'objet générique : maquette numérique BIM (incluant les différentes représentations vues précédemment). Interpréter la construction de cet objet dans sa globalité peut donc être entendu comme le produit d'interactions entre différents corps professionnels : l'architecte contrôle sur la maquette métier que les chemins de câble de l'électricien restent dans le gabarit du plénum; l'ingénieur structure base ses calculs sur le modèle géométrique de l'entreprise de gros-œuvre; l'équipe de synthèse analyse les maquettes métiers des différentes entreprises et émet des alertes sur les clashs ${ }^{17}$ ou les problèmes d'interfaces qu'elle constate; les livrables sont extraits des différentes maquettes métiers et sont vérifiées par la maitrise d'œuvre pour délivrer ses VISA; le BIM manager assiste, à distance, les acteurs qui rencontrent des difficultés techniques avec leurs propres maquettes métiers et s'assure que la charte BIM soit respectée. Toutes ces nouvelles interactions ont d'ailleurs tendance à redistribuer les connaissances tout en horizontalisant les rapports professionnels, ce qui entraine certainement une recomposition de l'autorité de l'architecte. La mise en place d'un projet BIM semble ainsi avoir tendance à regrouper les différents acteurs (architectes, ingénieurs, entrepreneurs, économistes, BIM managers, maîtres d'ouvrages) issus de mondes sociaux différenciés, autour de la construction collective d'un objet-frontière incarné par la maquette numérique.

Une démarche parallèle consisterait à identifier et évaluer le temps, ou la période pendant laquelle l'objet considéré est construit, échangé et utilisé par les différents acteurs. Sur cette question reprenons la comparaison avec la maquette physique initialement considérée. Premièrement, la maquette physique peut, elle aussi, être perçue comme un objet-frontière sur lequel s'appuient nombre d'échanges et négociations inter-acteurs. En revanche, son utilisation se restreint bien souvent à la phase de conception et il reste rare que les architectes réalisent des maquettes physiques dès lors que le chantier a débuté. De plus, lorsque le bâtiment est livré, la maquette physique n'est généralement là que comme

\footnotetext{
14 Système d'Information Géographique.

${ }^{15}$ Chauffage, Ventilation, Climatisation.

${ }^{16}$ Fiche Modificative de Travaux.

${ }^{17}$ Un clash représente une collision entre deux maquettes numériques (entendues ici comme modèles géométriques sémantiques).
} 
une relique ou le témoin d'un geste ou d'une activité passée. En revanche concernant la maquette numérique BIM : la période de son élaboration, d'échange et d'utilisation de l'objet surpasse largement l'unique phase de conception. En effet, il nous faut admettre que les objectifs de ce nouveau type de maquette ne peuvent se restreindre uniquement à des visées de conception. L'objet doit effectivement assister et permettre la construction du bâtiment, mais aussi faciliter la gestion et l'exploitation-maintenance de l'édifice construit. Ainsi la maquette numérique BIM s'ancre dans une nouvelle temporalité qui ne se circonscrit plus à la phase de conception privilégiée de l'architecte. On peut donc supposer que les nouvelles porosités que l'objet entretient (et doit entretenir) avec les phases de construction et d'exploitation-maintenance pourraient avoir, par effet boomerang, des incidences importantes sur la programmation et la conception et donc sur l'activité de l'architecte.

Enfin, il convient aussi de noter que la maquette numérique semble progressivement répondre à des enjeux (d'usages et temporels) assez similaires à la construction du bâtiment physique. Ne peut-on d'ailleurs pas considérer que l'objet finalisé d'une maquette numérique BIM n'est autre que le double du bâtiment physique : à savoir un bâtiment numérique ? Et si le bâtiment n'est pas un « objet statique, mais plutôt un projet en mouvement », car il continue d'être transformé par ses usagers (Latour et Yaneva, 2008, p. 81), nous ne sommes pas loin de penser que la construction de la maquette numérique vient superposer un nouveau projet au projet : construire un bâtiment numérique en parallèle d'un bâtiment physique. Dans ces conditions, se pourrait-il que la maquette numérique BIM et ce nouveau projet ne fassent qu'un?

\section{Conclusion}

La mise en parallèle de la maquette numérique avec la maquette physique nous a permis d'amorcer une recomposition des objets techniques que mobilise l'architecte. On observera aussi que l'évolution des outils traditionnels de l'architecte conditionne son rapport aux autres professions. En effet, si la maquette physique (tout comme le plan ou la coupe) était jusqu'alors l'incarnation d'un savoir-faire et donc d'une expertise identifiée, la maquette numérique BIM (condensant en partie les fonctionnalités des anciens outils de l'architecte) devient en revanche un objet distribué entre tous les acteurs du projet. Elle ne constitue donc plus l'outil exclusif de l'architecte, le dépossédant ainsi probablement d'une certaine partie de son expertise et donc de son pouvoir. La maquette numérique BIM semble aussi définir un tout autre type de maquette, aux frontières plus larges et encore mouvantes, qu'il n'est plus possible de confondre avec une maquette physique de conception. Pour mieux comprendre l'objet, il nous faut donc continuer d'étudier les effets qu'induit ce nouvel artefact sur les pratiques professionnelles et le déroulement du projet. L'ANT semble alors proposer un cadre théorique pertinent à cette investigation et l'introduction du concept d'objet-frontière nous apparaît être un levier capable de mettre en relief les nouveaux nœuds et controverses que fait émerger le mode projet BIM. D'ailleurs, si l'ANT a déjà été utilisée autour de différentes questions liées à l'architecture (Callon, 1996 ; Latour et Yaneva, 2008 ; Yaneva 2009), son application au contexte spécifique des projets BIM reste encore rare (Linderoth, 2009 ; Sørensen, Frandsen, et Øien, 2015 ; Teulier, 2017) et les études portant sur la construction des maquettes numériques BIM encore à faire. Enfin, si la maquette physique semblait se restreindre à la phase de conception ou aux moments de présentations au client, la maquette numérique traverse, elle, l'ensemble des phases du projet depuis la phase de programmation jusqu'à l'exploitation-maintenance. Elle suit le projet du bâtiment physique au plus près, en construisant un bâtiment numérique qui lui est rattaché. Cet entrelacement entre la conception, la construction puis la gestion de ces deux objets : le bâtiment physique et le bâtiment numérique, conduit à l'hypothèse que la 
maquette numérique pourrait finalement définir un nouveau type projet, venant se coupler au traditionnel projet architectural, mais dont la nature et les visées demeurent exclusivement numériques. Il conviendrait donc d'interroger à une échelle plus large les perspectives théoriques que semble dessiner le mode projet BIM. En effet, la construction de bâtiments et de la ville passe dorénavant par une numérisation des pratiques, des représentations, mais aussi des usages et de la gestion même des édifices réalisés. Tenter de décrire la construction de nouveaux artefacts numériques tels que la maquette BIM doit aussi être un moyen de questionner les objectifs que se donnent les architectes. À n'en pas douter, les architectes construisent déjà les premières briques physiques et numériques de la fameuse smartcity.

\section{Bibliographie}

Akrich, M. (1987). Comment décrire les objets techniques ? Techniques et culture, 49-64. Éditions de la maison des sciences de l'homme.

Alberti, L.B. (2004). L'art d'édifier. Traduit par Pierre Caye et Françoise Choay. Sources du savoir. Paris : Seuil.

Bassereau, J.-F., Charvet Pello, R., Faucheu, J., Delafosse D. (2015). Les objets intermédiaires de conception/design, instruments d'une recherche par le design. Science $d u$ Design, 2, 48-63.

Boujut, J-F., et Blanco, E. (2003). Intermediary Objects as a Means to Foster Co-operation in Engineering Design. Computer Supported Cooperative Work, 12, 205-219.

Callon, M. (1996). Le travail de la conception en architecture. In Situations, (p. 25-35) Les cahiers de la recherche architecturale 37. Marseille : Ed. Parenthèses.

Chalon, R. (2004). Réalité mixte et travail collaboratif: IRVO, un modèle de l'interaction homme-machine. École Centrale de Lyon.

Coulais, J-F. (2015). Images virtuelles et horizons du regard. Visibilités calculées dans l'histoire des représentations. Genève : Métis Presses.

Deshayes, C. (2013). Aspects dialogiques de l'esquisse architecturale 2D ou 3D. In Echelles, Espaces, Temps, par Zreik, K., et Yacoub, C., Europia, (pp. 117-133). Paris : Europia.

Estevez, D. (2014a). Dialogue entre maquette et modèle en architecture. In Les modèles, possibilités et limites: jusqu'où va le réel ? Jean-Michel Levy, (pp. 77-86). Paris : Editions Matériologiques.

Estevez, D. (2014b). Petite mythologie des maquettes. Architecture d'Aujourd'hui, décembre 2014.

Girard, C. (2014). L'architecture, une dissimulation. La fin de l'architecture fictionnelle à l'ère de la simulation intégrale. In Modéliser et simuler - Tome 2, par Varenne, F., Silberstein, M., Dutreuil, S., Huneman, P., Bernaud, J-L. et Lhotellier, L. Paris : Editions Matériologiques.

Halin, G., et Gallas, M-A. (2016). Une approche pédagogique par les modèles pour la sensibilisation au concept de BIM (Maquette Numérique). In SCAN'16 : Séminaire de Conception Architecturale Numérique, par Goulette, J-P. et Ferries, B., (pp. 85-96). Toulouse.

Jeantet A. (1998). Les objets intermédiaires dans la conception. Éléments pour une sociologie des processus de conception. Sociologie du travail, 40-3, (pp. 291-316)

Latour, B. (1992). Aramis ou l'amour des techniques. Textes à l'appui Série anthropologie des sciences et des techniques. Paris: Éd. la Découverte.

Latour, B., et Yaneva A. (2008). Give me a gun and I will make all buildings move: an ANT's view of architecture. In Explorations in Architecture : Teaching, Desing, Research, par Reto Geiser, (pp. 80-89). Basel : Birkhäuser. 
Lefauconnier, M. (2017). Le BIM face au droit. In Le BIM éclairé par la recherche, par Riss, S., Talon, A., et Teulier, R., (pp. 91-100). Paris: Eyrolles.

Linderoth, H.C.J. (2009). Understanding adoption and use of BIM as the creation of actor networks. Automation in Construction 19 (pp. 66-72).

Loyer, F. (1974). Pour bien lire une maquette d'architecture: le relatoscope. Communication et langages 23 (1) (pp. 56-75).

MacEachren, A.M. (2001). Cartography and GIS : Extending Collaborative Tools to Support Virtual Teams. Progress in Human Geography 25 (3) (pp. 431-444).

Sørensen, N. L., Frandsen, A. K. et Borgestrand Øien T. (2015). Architectural Competitions and BIM. Procedia Economics and Finance 21: (pp.239-246).

Star, S. L., et Griesemer. J.R. (1989). Institutionnal Ecology, "Translation”, and Boundary Objects : Amateurs and Professionals in Berkeley's Museum of Vertebrate Zoology". Social Studies of Science 19 (3) : (pp.387-420).

Star, S-L. (2010). Ceci n'est pas un objet-frontière ! Réflexions sur l'origine d'un concept. Revue d'anthropologie des connaissances. 4-1, (pp. 18-35).

Teulier, R. (2017). La coopération : processus fondamentaux et implications pour le travail collaboratif dans une démarche BIM. In S. Riss, A. Talon, R. Teulier, Le BIM éclairé par la recherche, (pp. 59-74). Paris : Eyrolles.

Vinck, D. (2009). De l'objet intermédiaire à l'objet-frontière. Vers la prise en compte du travail d'équipement. Revue d'anthroplogie des connaissances 3 (1) (pp. 51-72).

Yaneva, A. (2009). Made by the Office for Metropolitan Architecture : An Ethnography of Design. Rotterdam : 010 Publishers. 